$\Rightarrow$ BACTERIAL PATHOGENICITY

\title{
A competent escape for Listeria
}

The facultative intracellular pathogen Listeria monocytogenes must escape from the phagosome into the host cell cytosol to complete the infectious cycle, and phagosomal escape is therefore an important component of virulence. Rabinovich et al. now reveal that the L. monocytogenes competence (Com) system has a key role in phagosomal escape and that activation of this system is controlled by a prophage excision event.

The term competence describes the physiological state in which bacteria can undergo transformation (the uptake of exogenous DNA). The genes of the Com system, including the late comG, comE and comF operons, and the main regulators have been characterized in Bacillus subtilis. Although L. monocytogenes contains homologues of most of the late com genes, it is not transformable, and the only known regulator in this species is a homologue of the key B. subtilis activator, ComK.

In several $L$. monocytogenes strains, the comK gene is disrupted by a temperate prophage; therefore, the impact of this insertion on virulence was investigated. In L. monocytogenes str. $10403 S$, comK is interrupted by the $\varphi 10403$ S prophage. Whole-genome transcriptomics of this strain growing in bonemarrow-derived macrophages, combined with analysis of the growth effects of in-frame com deletions, revealed that the comG and comE operons are required for intracellular growth. Further mutant analysis showed that only the membrane channel, encoded by comEC, and the pseudopilus, encoded by the comG operon, were required, suggesting that involvement of the Com system is independent of DNA uptake. The mutant analysis also revealed that the comEC and comG mutants were impaired in phagosomal escape, and this was confirmed by fluorescence microscopy.

How is the Com system regulated? The authors examined the promoters of the three com operons and identified a conserved binding site for the ComK activator. By monitoring the fate of comK during growth in liquid culture, they found that comK was disrupted in exponential phase but intact in stationary phase, suggesting that the $\varphi 10403 S$ prophage had been excised. Similarly, during intracellular growth, comK was intact and an excised form of the phage genome could be detected. There was no evidence of bacterial cell lysis or progeny virion production, however, suggesting that phage propagation is blocked. Finally, by assessing the growth of a range of deletion mutants, the authors established that an intact comK gene (and, hence, prophage excision) is required for intracellular growth.

The authors propose that prophage excision during phagocytosis generates an intact comK gene, and the resultant functional ComK protein activates the Com system to induce phagosomal escape. Further work is required to determine what triggers prophage excision and the exact mechanism by which the Com pseudopilus and membrane channel promote phagosomal escape.

Sheilagh Molloy

ORIGINAL RESEARCH PAPER Rabinovich, L. et al. Prophage excision activates Listeria competence genes that promote phagosomal escape and virulence. Cell 150 , 792-802 (2012) 